\title{
Thermoresponsive Interplay of Water Insoluble Poly(2-alkyl-2-oxazoline)s Composition and Supramolecular Host-Guest Interactions
}

\section{Victor R. de la Rosa ${ }^{1}$, Werner M. Nau ${ }^{2}$ and Richard Hoogenboom ${ }^{1, *}$}

1 Supramolecular Chemistry Group, Department of Organic and Macromolecular Chemistry, Ghent University, Krijgslaan 281 S4, 9000 Ghent, Belgium;

E-Mail: victor.retamerodelarosa@ugent.be

2 Department of Life Sciences and Chemistry, Jacobs University Bremen, Campus Ring 1, D 28759 Bremen, Germany; E-Mail: w.nau@jacobs-university.de

* Author to whom correspondence should be addressed; E-Mail: richard.hoogenboom@ugent.be; Tel.: +32-926-444-82.

Academic Editors: Graeme Cooke and Patrice Woisel

Received: 9 March 2015 / Accepted: 27 March 2015 / Published: 2 April 2015

\begin{abstract}
A series of water insoluble poly[(2-ethyl-2-oxazoline)-ran-(2-nonyl-2-oxazoline)] amphiphilic copolymers was synthesized and their solubility properties in the presence of different supramolecular host molecules were investigated. The resulting polymer-cavitand assemblies exhibited a thermoresponsive behavior that could be modulated by variation of the copolymer composition and length. Interestingly, the large number of hydrophobic nonyl units across the polymer chain induced the formation of kinetically-trapped nanoparticles in solution. These nanoparticles further agglomerate into larger aggregates at a temperature that is dependent on the polymer composition and the cavitand type and concentration. The present research expands the understanding on the supramolecular interactions between water insoluble copolymers and supramolecular host molecules.
\end{abstract}

Keywords: Lower Critical Solution Temperature (LCST); temperature responsive polymers; poly(2-alkyl-2-oxazoline)s; cyclodextrins; cucurbit[7]uril; supramolecular interactions; nanoparticles 


\section{Introduction}

Thermoresponsive polymers, able to respond to changes in the environmental temperature, constitute a fascinating polymer class that has resulted in a myriad of applications in fields as diverse as construction [1,2], water management [3], separation sciences [4,5], or shape memory materials [6]. In solution, thermoresponsive polymers result in the so called smart fluids or smart soluble materials, which exhibit parallels with natural responsive systems [7]. In this context, thermoresponsive polymers have become an invaluable tool to develop new applications in biomedicine [8], especially in drug and gene delivery [9], tissue engineering [10] and as sensors [11,12].

In contrast to their natural counterparts that usually exhibit an upper critical solution temperature (UCST), most synthetic thermoresponsive polymers exhibit a lower critical solution temperature (LCST) behavior, which arises from their negative entropy of solvation. This involves a sharp polymer phase separation upon heating the solution above a specific temperature, a feature that has been successfully exploited in the development of soluble temperature sensors that operate at the nanoscale [12-16]. The polymer transition temperature depends upon polymer molecular mass, composition and concentration and is regarded as cloud point temperature $\left(\mathrm{T}_{\mathrm{CP}}\right)$. Controlling the hydrophobicity of the polymer thus allows the tuning of the $\mathrm{T}_{\mathrm{CP}}$ to tailor it to the required application, as has been extensively investigated by varying the polymer composition [17], e.g., in poly(oligo ethylene glycol (meth)acrylate)s [18], poly(2-hydroxypropyl acrylate)s [19], and poly(2-oxazoline)s [20-25]. In particular, copolymerization of hydrophobic 2-nonyl-2-oxazoline (NonOx) and hydrophilic 2-ethyl-2-oxazoline (EtOx) yields random copolymers whose thermal responsiveness can be easily tuned by the ratio of both monomers [26,27]. In addition, the polymer hydrophilic-hydrophobic balance can be controlled by specific supramolecular interactions with amphiphilic host molecules, as has been found in end-group modified poly(2-alkyl-2-oxazoline)s (PAOx) and in other polymer platforms [28-32]. Cavitands can form complexes with matching guest units along the polymer chain offering control on the ensemble's transition temperature [33] and morphology [34-39] in solution, as has also been investigated for crown-ether decorated polymers [40,41].

Recently, we have studied the influence of a range of different supramolecular hosts on the solubility properties of a thermoresponsive PEtOx-ran-PNonOx random copolymer containing 12 mol \% nonyl side chains, wherein the nonyl chains act as guest units for the cavitands [42]. The cavitands tested were selected among well-known hosts for aliphatic chains: cucurbit[7]uril (CB7), alpha-cyclodextrin ( $\alpha \mathrm{CD})$, and hydroxypropylated alpha- and beta-cyclodextrins ( $\mathrm{HP} \alpha \mathrm{CD}$ and $\mathrm{HP} \beta \mathrm{CD}$, respectively). Besides obtaining an unprecedentedly broad tunability of the $\mathrm{T}_{\mathrm{CP}}$ of $c a .30 \mathrm{~K}$ with stoichiometric amounts of cavitand, the extent of the transition was found to be directly correlated to the polymer-cavitand host-guest binding affinity. Indeed, the power of each cavitand to increase the $\mathrm{T}_{\mathrm{CP}}$ of the polymer-cavitand ensemble followed the order of nonyl-cavitand binding affinity, i.e., $\mathrm{CB} 7\left(\mathrm{~K}_{\mathrm{a}} \approx 2200 \mathrm{M}^{-1}\right)$ $>>\alpha \mathrm{CD}\left(\mathrm{K}_{\mathrm{a}} \approx 440 \mathrm{M}^{-1}\right)>\operatorname{HP} \alpha \mathrm{CD}\left(\mathrm{K}_{\mathrm{a}} \approx 220 \mathrm{M}^{-1}\right)>\operatorname{HP} \beta \mathrm{CD}\left(\mathrm{K}_{\mathrm{a}} \approx 120 \mathrm{M}^{-1}\right)$ [42]. In a second report, we demonstrated that fully water-insoluble PEtOx-ran-PNonOx copolymers containing $33 \mathrm{~mol} \%$ NonOx assemble into defined nanoparticles in presence of excess cavitands. Interestingly, these nanoparticles either reversibly or irreversibly agglomerated upon heating enabling their use as temperature sensors with rewritable or permanent memory functions, respectively, depending on the utilized cavitands [43]. 
In the present study, we have investigated the effect of increasing the polymer length and nonyl side chains content on the thermoresponsive behavior of the polymer-cavitand ensembles in further detail. All the here reported PEtOx-ran-PNonOx copolymers were insoluble in water at near $0{ }^{\circ} \mathrm{C}$. The copolymers could however be brought in solution in the presence of the cavitands, leading to thermoresponsive polymer-cavitand ensembles (see Figure 1).

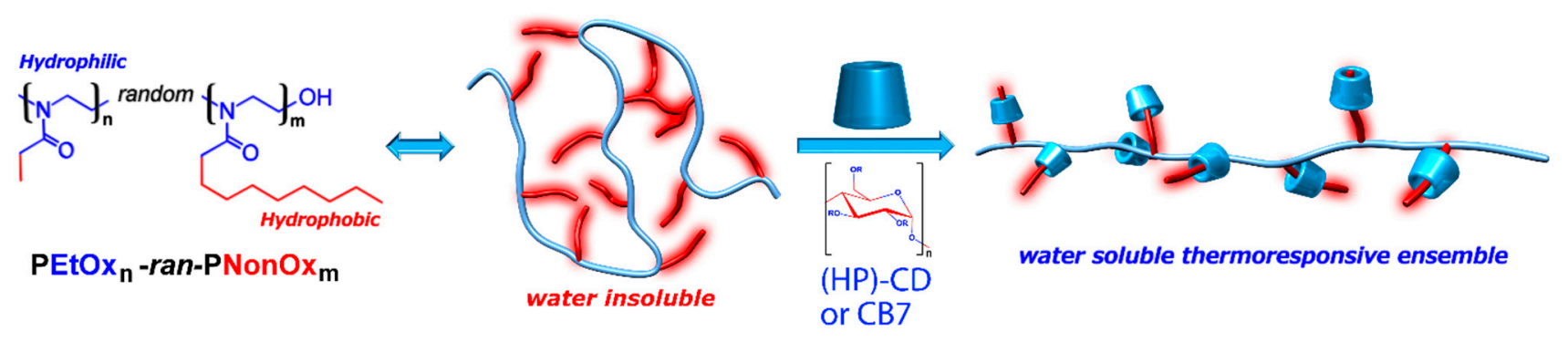

Figure 1. A series of amphiphilic PEtOx-ran-PNonOx random copolymers was synthesized and their solubility properties were studied in the presence of a range of different supramolecular host molecules. The picture describes the supramolecular complexation of a PEtOx-ran-PNonOx random copolymer with cavitands resulting in the formation of thermoresponsive supramolecular complexes.

Interestingly, increasing the number of nonyl guest units, and consequently the strength of the polymer-polymer interactions, led to a dependence of the $\mathrm{T}_{\mathrm{CP}}$ on the cavitand hydrophilicity, and not only on the strength of the nonyl-cavitand host-guest interactions. Hereby we present a detailed study on the differential interplay between supramolecular hosts and a range of water insoluble PEtOx-ran-PNonOx copolymers, aiming to expand the understanding on the supramolecular interactions of host molecules with water insoluble polymers.

\section{Results and Discussion}

The soluble copolymer that was studied in our previous report [42] contained $12 \%$ nonyl side chains, and a composition of PEtOx90-ran-PNonOx12 (nearly 100 repeating units). This copolymer exhibited a $\mathrm{T}_{\mathrm{CP}}$ of $\mathrm{ca} .10^{\circ} \mathrm{C}$. In the present study, we aimed to analyze the differential interaction of supramolecular hosts with highly hydrophobic PEtOx-ran-PNonOx random copolymers, investigating the effect of polymer composition and length. To this end, a series of PEtOx-ran-PNonOx random copolymers with increasing NonOx content and varying degree of polymerization was synthesized.

\subsection{Poly(2-ethyl-2-oxazoline)-ran-poly(2-nonyl-2-oxazoline) Synthesis}

The copolymerizations were performed following a previously reported protocol [26] by preparing solutions of EtOx and NonOx with the desired stoichiometry in dry acetonitrile, and a total monomer concentration of $4 \mathrm{M}$. The initiator used was methyl tosylate (MeOTs) [44] and the monomer to initiator ratio was selected to obtain copolymers with the desired length (details are given in the Experimental Section). All polymerizations were performed to up to full conversion in capped vials in a microwave synthesizer at $140^{\circ} \mathrm{C}$ and terminated with methanolic $\mathrm{KOH}$, resulting in hydroxyl-terminated polymers (see Figure 2). 


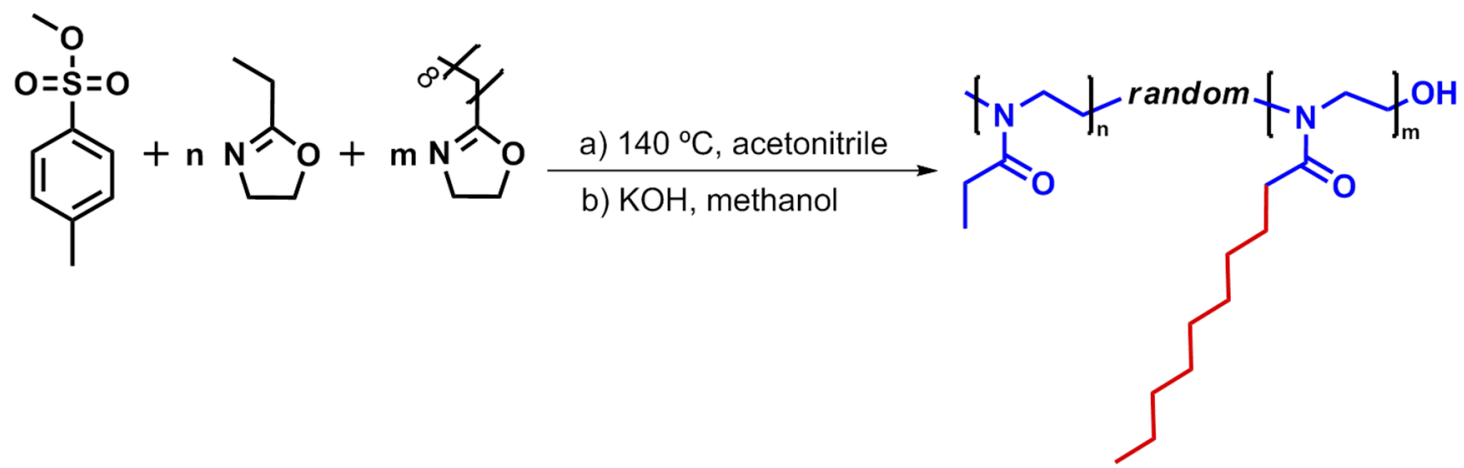

Figure 2. Schematic representation of the living cationic ring-opening copolymerization of 2-ethyl-2-oxazoline (EtOx) and 2-nonyl-2-oxazoline (NonOx) initiated by methyl tosylate resulting in PEtOx-ran-PNonOx random copolymers.

Interestingly, the EtOx-NonOx statistical copolymerization results in ideal random copolymer structures, since the reactivity ratios of both monomers are equal to unity, as has been previously reported $[26,45]$. The obtained $\left.\mathrm{P}\left[(\mathrm{EtOx})_{n} \text {-ran-(NonOx }\right)_{m}\right]$ copolymer compositions were determined by ${ }^{1} \mathrm{H}-\mathrm{NMR}$ spectroscopy (see Figure 3 and Table 1).
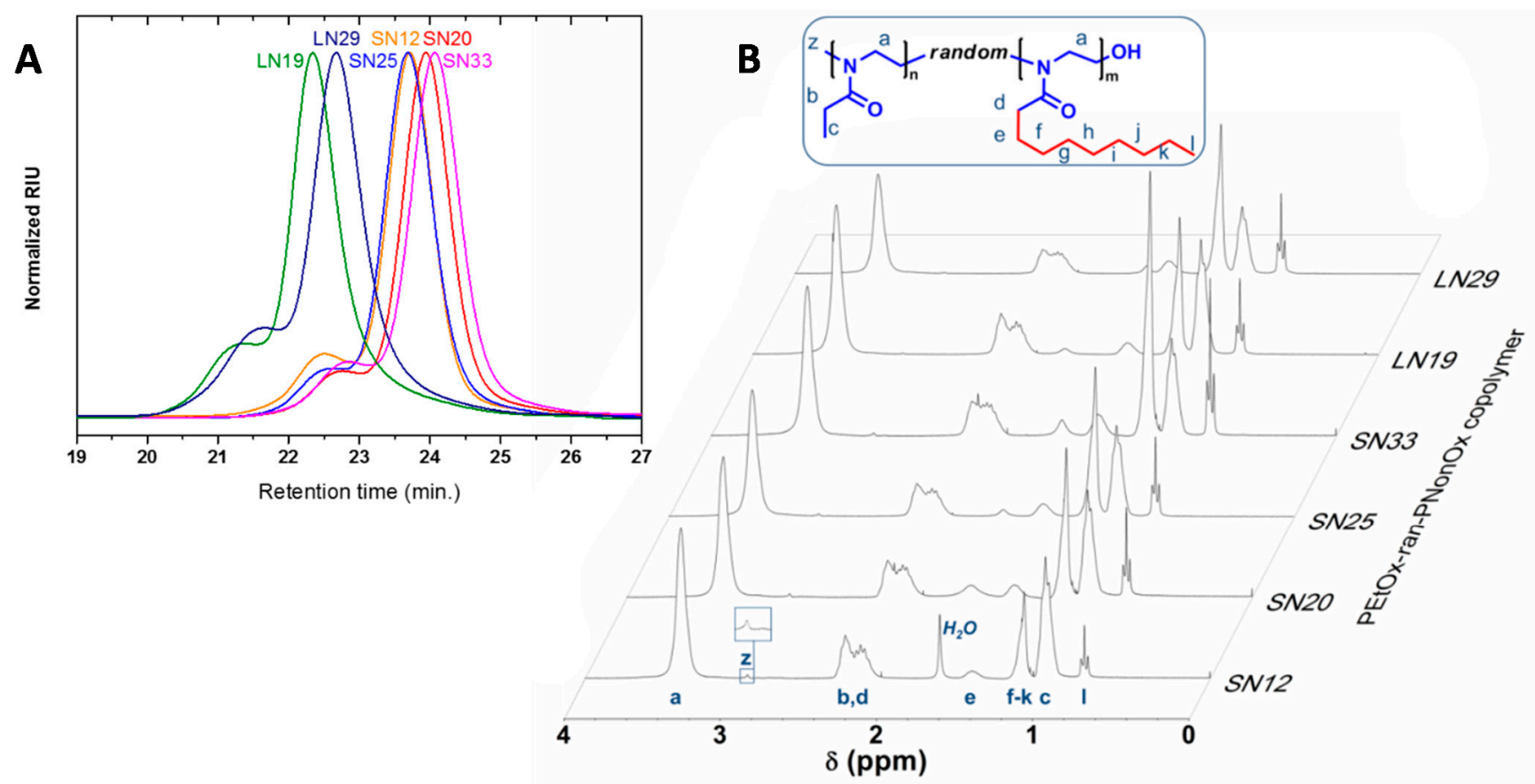

Figure 3. (A) Size exclusion chromatography (SEC) data of the synthesized $\mathrm{PEtOx}_{n}$-ran-PNonOx $m$ random copolymers. Albeit a high molecular weight distribution is observed as shoulder in all cases, ascribed to chain coupling, well-defined polymers with dispersities around 1.1 were obtained. SEC eluent: $N, N$-dimethylacetamide containing $50 \mathrm{mM} \mathrm{LiCl}$; (B) ${ }^{1} \mathrm{H}-\mathrm{NMR}$ spectra of the investigated PEtOx-ran-PNonOx random copolymers in $\mathrm{CDCl}_{3}$. Legend: $\mathrm{S}=$ Short polymer, $\mathrm{DP} \approx 100, \mathrm{~L}=$ Long polymer $\mathrm{DP} \approx 200$. $\mathrm{N}=\%$ Nonyl side chains in number. 
Table 1. Composition and size exclusion chromatography (SEC) data of the synthesized $\mathrm{PEtOx}_{n}$-ran-PNonOx $m$ random copolymers. Polymer composition was calculated by ${ }^{1} \mathrm{H}-\mathrm{NMR}$ analysis. SEC eluent: $N, N$-dimethylacetamide containing $50 \mathrm{mM}$ LiCl. Calibrated against PMMA standards. Polymer ID legend: $\mathrm{S}=$ Short polymer, DP $\approx 100, \mathrm{~L}=$ Long polymer $\mathrm{DP} \approx 200$.

\begin{tabular}{|c|c|c|c|c|c|}
\hline \multirow{2}{*}{ ID } & \multirow{2}{*}{ Polymer Composition } & \multirow{2}{*}{ DP } & \multirow{2}{*}{$\begin{array}{l}\% \text { NonOx } \\
\text { (Number) }\end{array}$} & \multicolumn{2}{|c|}{ SEC } \\
\hline & & & & $\mathbf{M}_{\mathrm{n}}$ & Ð \\
\hline SN12 & $\mathrm{PEtOx}_{90}-$ ran-PNonOx 12 & 102 & 12 & 18,000 & 1.08 \\
\hline SN20 & $\mathrm{PEtOx}_{84}-r a n-\mathrm{PNonOx}_{21}$ & 105 & 20 & 17,500 & 1.08 \\
\hline SN25 & $\mathrm{PEtOx}_{75}$-ran-PNonOx 25 & 100 & 25 & 16,500 & 1.07 \\
\hline SN33 & $\mathrm{PEtOx}_{62}-r a n-\mathrm{PNonOx}_{29}$ & 91 & 33 & 15,000 & 1.09 \\
\hline LN19 & $\mathrm{PEtOx}_{162}$-ran-PNonOx 38 & 200 & 19 & 29,700 & 1.11 \\
\hline LN29 & $\mathrm{PEtOx}_{140}$-ran-PNonOx 57 & 197 & 29 & 28,000 & 1.13 \\
\hline
\end{tabular}

All the synthesized copolymers exhibited dispersities below 1.15, as determined by size exclusion chromatography, indicating good control over the polymerizations (see Figure 3). Nevertheless, as commonly observed, a high molecular weight shoulder was present in all copolymers, presumably due to chain-transfer reactions [46]. The observed differences in retention time are not only ascribed to different number average molecular weight, but also to the different hydrophobicity of the copolymers. As the content of NonOx in the copolymer increases, so does its hydrophobicity, resulting in a less efficient solvation by the polar $N, N$-dimethylacetamide eluent, producing a smaller hydrodynamic volume. Consequently, prolonged retention times are observed for the most hydrophobic copolymers. This effect is especially noticeable for the LN19 and LN29 copolymers, both with a degree of polymerization (DP) close to 200 repeating units; albeit LN29 has a higher molecular weight, its retention time is higher than that of LN19, indicating a distinctly larger hydrophobicity. Later on, these preliminary observations will translate into marked differences in the copolymer solubility properties in the presence of cavitands.

\subsection{Influence of Nonyl Side Chain Content: Poly(2-ethyl-2-oxazoline)-ran-poly(2-nonyl-2-oxazoline)} Containing 20\% Nonyl Chains

Initially, we investigated a PEtOx84-ran-PNonOx 21 copolymer containing $20 \%$ nonyl chains in number, therefore roughly doubling the NonOx content of the previously reported PEtOx 90 -ran-PNonOx 12 copolymer, while maintaining its length at $c a .100$ repeating units. The additional NonOx content made this copolymer insoluble in water, and therefore the addition of host molecules to the solution, followed by freezing and thawing under sonication in an ice-bath, was necessary to bring the copolymer in solution. This protocol seemed to favor the formation of inclusion complexes with the nonyl chains affording the solubilization of the copolymer.

A $5 \mathrm{mg} \cdot \mathrm{mL}^{-1}$ solution of the $\mathrm{PEtOx} 84$-ran-PNonOx 21 random copolymer was prepared and titrated with different cavitands, while monitoring the evolution of the copolymer $\mathrm{T}_{\mathrm{CP}}$. The cavitands that previously exhibited a stronger binding constant with nonyl chains were selected to perform these studies, namely $\mathrm{CB} 7, \alpha \mathrm{CD}$, and $\mathrm{HP} \alpha \mathrm{CD}$. Interestingly, the hydrophilicity order of these cavitands is the inverse of their binding affinity to nonyl chains, i.e., $\mathrm{HP} \alpha \mathrm{CD}>>\alpha \mathrm{CD}>\mathrm{CB}$, with water solubilities that 
are, respectively, $>600,145$ and $39 \mathrm{mg} \cdot \mathrm{mL}^{-1}[47,48]$. To be able to titrate $\mathrm{CB} 7$, due to its relatively low solubility, a $2 \mathrm{mg} \cdot \mathrm{mL}^{-1}$ solution of the copolymer was used. As previously found for the PEtOx-ran-PNonOx containing 12\% NonOx, the temperature-triggered phase transition of all solutions could be tuned by the addition of the cavitands (see Figure 4).

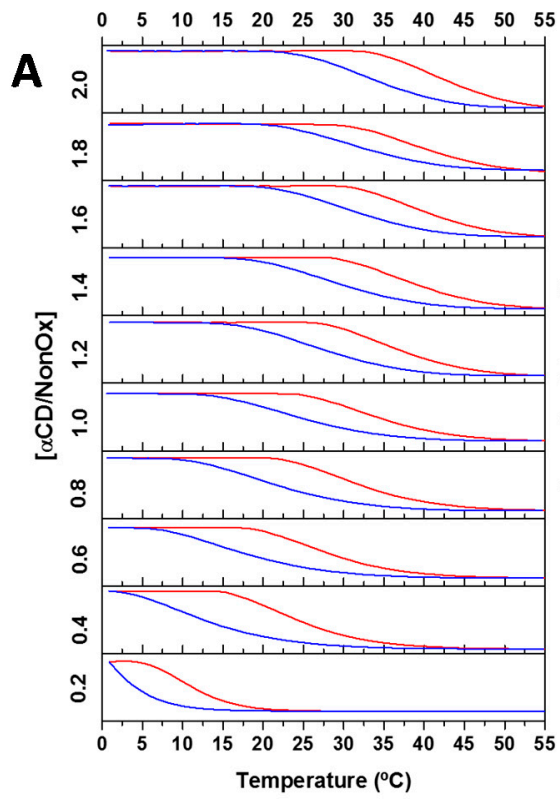

$$
\text { PEtOx }_{84} \text {-ran-PNonOx } 21
$$

$\begin{array}{llllllllllll}0 & 5 & 10 & 15 & 20 & 25 & 30 & 35 & 40 & 45 & 50 & 55\end{array}$
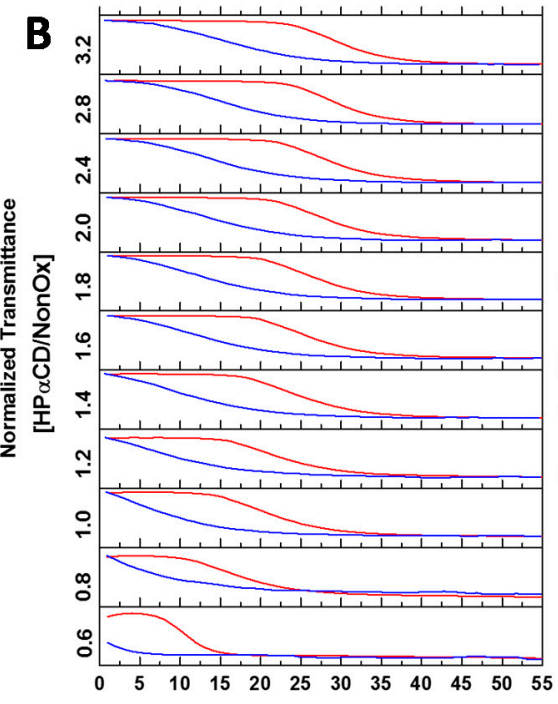

Temperature $\left({ }^{\circ} \mathrm{C}\right)$

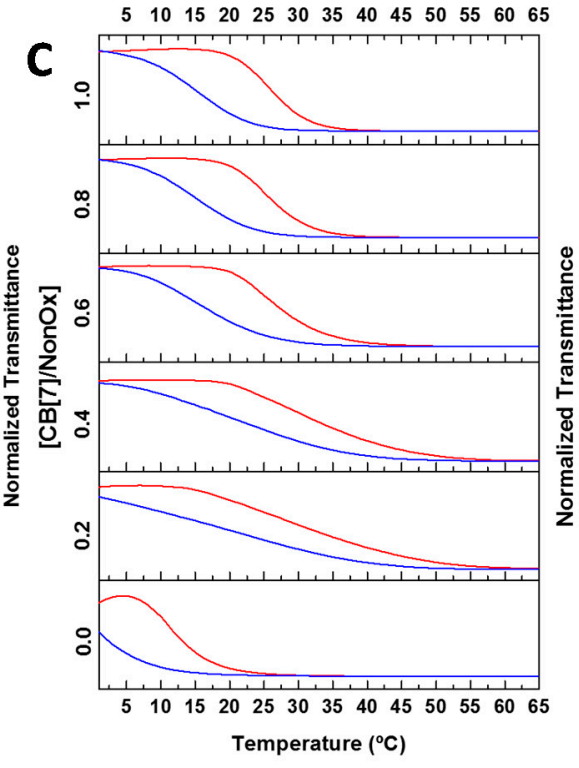

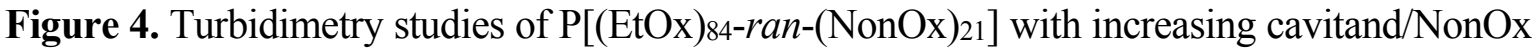
molar ratios. $5 \mathrm{mg} \cdot \mathrm{mL}^{-1}$ solutions of copolymer were titrated with $\alpha \mathrm{CD}(\mathbf{A})$ and $\mathrm{HP} \alpha \mathrm{CD}(\mathbf{B})$, whereas a $2 \mathrm{mg} \cdot \mathrm{mL}^{-1}$ solution of copolymer was titrated with $\mathrm{CB} 7(\mathbf{C})$. The heating and cooling ramps are represented by the top (red) and bottom (blue) curves, respectively. Rate: $1 \mathrm{~K} \cdot \mathrm{min}^{-1}, \lambda=700 \mathrm{~nm}$.

However, instead of a sharp shift in transmittance, the transition is now much more progressive. Since the solubility phase-transition is produced by the disassembly of the host-guest complexes, the progressive transition observed here indicates slow kinetics in the breakage/formation of the cavitand-nonyl host-guest complexes. This can be explained by the larger hydrophobicity of the copolymer that forms kinetically-trapped nanoparticles entrapping the cavitands and partially isolating them from the surrounding aqueous solution. This hypothesis was supported by temperature-dependent dynamic light scattering (DLS) measurements of a solution containing the copolymer and 1.0 equivalent $\alpha \mathrm{CD}$, which showed the formation of well-defined nanoparticles beyond $c a .20{ }^{\circ} \mathrm{C}$. As seen in Figure 5, at low temperatures a single particle distribution of $c a .10 \mathrm{~nm}$ in size (PDI $=0.15)$ is observed. This is ascribed to the formation of well hydrated random-coil copolymer-cavitand ensembles, and is analogous to the behavior observed for the previously studied copolymer containing $12 \%$ NonOx. When this copolymer was heated beyond its $\mathrm{T}_{\mathrm{CP}}$ in the presence of 1.0 equivalent $\alpha \mathrm{CD}$, large $>1000 \mathrm{~nm}$ aggregates were formed, indicating de-threading of the $\alpha \mathrm{CD}$ units and the collapse of the polymer chains into thermodynamically stable aggregates (data not shown). However, in the case of the PEtOx84-ran-PNonOx21 copolymer, upon heating beyond the $\mathrm{T}_{\mathrm{CP}}$ at $c a .22{ }^{\circ} \mathrm{C}$, instead of large aggregates, the formation of moderate size well-defined nanoparticles is observed $(\approx 250 \mathrm{~nm}$, 
PDI $<0.15)$. The slow heating/cooling rate applied in the DLS experiments $\left(\approx 0.01 \mathrm{~K} \cdot \mathrm{min}^{-1}\right)$ provides sufficient time for the copolymer-cavitand ensembles to change their conformation and adapt to the progressive temperature-induced breakage of nonyl-cavitand inclusion complexes. The nanoparticles slowly increased in size with temperature, possibly due to the incorporation of additional copolymer chains during heating or due to further agglomeration of the initially formed nanoparticles.

It should also be noted that the apparent hysteresis observed in the turbidimetry measurements originates from the relatively fast heating/cooling rate of $1 \mathrm{~K} \cdot \mathrm{min}^{-1}$ applied, as it is not seen in the DLS experiments, were a much lower rate was applied.

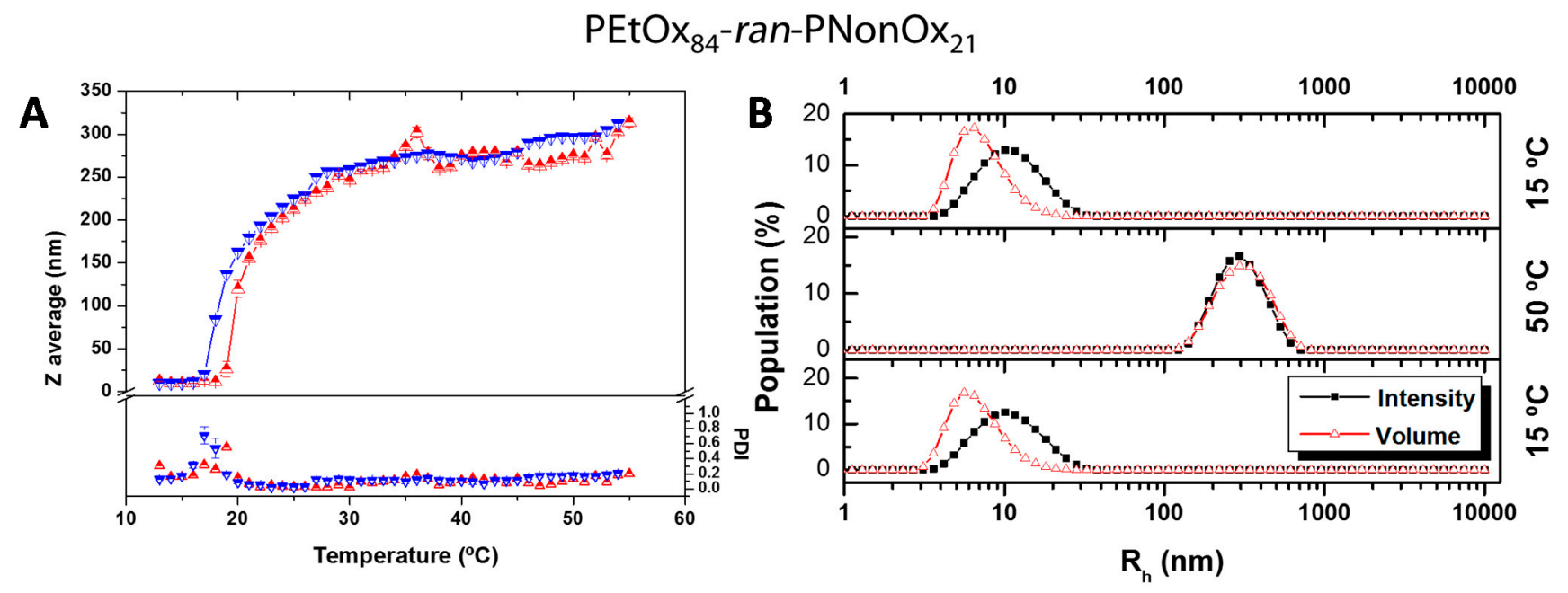

Figure 5. Temperature-dependent Dynamic Light Scattering (DLS) data for a $5 \mathrm{mg} \cdot \mathrm{mL}^{-1}$ solution of $\mathrm{P}\left[(\mathrm{EtOx})_{84}-\mathrm{ran}-(\mathrm{NonOx})_{21}\right]$ and 1.0 equivalent of $\alpha \mathrm{CD}$ (relative to nonyl side chains). (A) Z average and PDI evolution with temperature. > heating; Icooling. Random coil copolymer-cavitand ensembles are formed at low temperature and are stable up to $\approx 20{ }^{\circ} \mathrm{C}$ (transparent solution); above this temperature, the copolymer-cavitand ensembles re-arrange into well-defined nanoparticles $\left(279 \mathrm{~nm}\right.$, PDI $=0.090$ at $\left.50{ }^{\circ} \mathrm{C}\right)$. Four measurements are averaged at every temperature. Approximate heating/cooling rate: $\approx 0.01 \mathrm{~K} \cdot \mathrm{min}^{-1} ;$ (B) Particle size distribution of the sample at $15,50{ }^{\circ} \mathrm{C}$ and back to $15{ }^{\circ} \mathrm{C}$. At $15{ }^{\circ} \mathrm{C}$ individual copolymer-cavitand ensembles are observed. Heating beyond $20^{\circ} \mathrm{C}$ leads to the formation of well-defined nanoparticles that slowly grow with temperature $(279 \mathrm{~nm}$, PDI $=0.090$ at $50{ }^{\circ} \mathrm{C}$ ). Back to $15{ }^{\circ} \mathrm{C}$, the host-guest complexes re-form and random coil copolymer ensembles are observed $(10 \mathrm{~nm}, \mathrm{PDI}=0.136)$.

The titration with $\mathrm{HP} \alpha \mathrm{CD}$ yielded markedly different results, as 0.6 equivalents of $\mathrm{HP} \alpha \mathrm{CD}$ were necessary to solubilize the copolymer at near-zero degrees, in contrast to only 0.2 equivalents of $\alpha \mathrm{CD}$. Although $\mathrm{HP} \alpha \mathrm{CD}$ has a much larger hydrophilicity than native $\alpha \mathrm{CD}$, its lower binding affinity to nonyl chains results in a much lower ability to increase the solubility of the copolymer. On the other hand, the similar progressive decay in transmittance in the presence of $\mathrm{HP} \alpha \mathrm{CD}$ suggests the formation of kinetically-trapped structures as in the case of $\alpha \mathrm{CD}$. The low impact that increasing concentration of $\mathrm{HP} \alpha \mathrm{CD}$ has on the $\mathrm{T}_{\mathrm{CP}}$ indicates that a large number of nonyl chains is shielded from the surrounding aqueous environment, further supporting the formation of kinetically trapped nanoparticles with a hydrophobic core. 
On the other hand, $\mathrm{CB} 7$ produced an apparent large impact on the $\mathrm{T}_{\mathrm{CP}}$ at low $\mathrm{CB} 7$ concentrations but, overall, did not have a large effect on the copolymer $\mathrm{T}_{\mathrm{CP}}$. Temperature dependent DLS studies were performed in samples containing 0.2 and 1.0 equivalents of $\mathrm{CB} 7$ to investigate the reasons behind this behavior. The sample containing 0.2 equivalents of $\mathrm{CB} 7$ showed the absence of nanoparticle formation and the presence of large aggregates, even at low temperatures. This is manifested by the high PDI values observed below $20{ }^{\circ} \mathrm{C}$ by DLS (Figure 6). Possibly, the low hydrophilicity of the macromolecule constitutes an obstacle to disrupt the compact copolymer globules, which are stabilized by hydrophobic interactions among the nonyl side chains. Upon heating, macromolecular precipitates appear, that partially re-dissolve upon cooling below $20{ }^{\circ} \mathrm{C}$, resulting in an apparent increase in the $Z_{\text {ave. One }}$ equivalent of the cavitand was sufficient to form random coil copolymer ensembles of $\approx 9 \mathrm{~nm}$ in size at low temperatures, but these coexisted with aggregates with a variety of sizes (40-300 nm, PDI $\approx 0.8)$. Heating to temperatures above $20^{\circ} \mathrm{C}$ produced the collapse of the aggregates into large particles in the micrometer range. The presence of aggregates in solution, even at low temperatures, indicate the inability of CB7 to produce kinetically trapped nanoparticles, possibly due to its lower hydrophilicity compared to $\alpha \mathrm{CD}$ and $\mathrm{HP} \alpha \mathrm{CD}$.

0.2 Eq. $C B[7]$

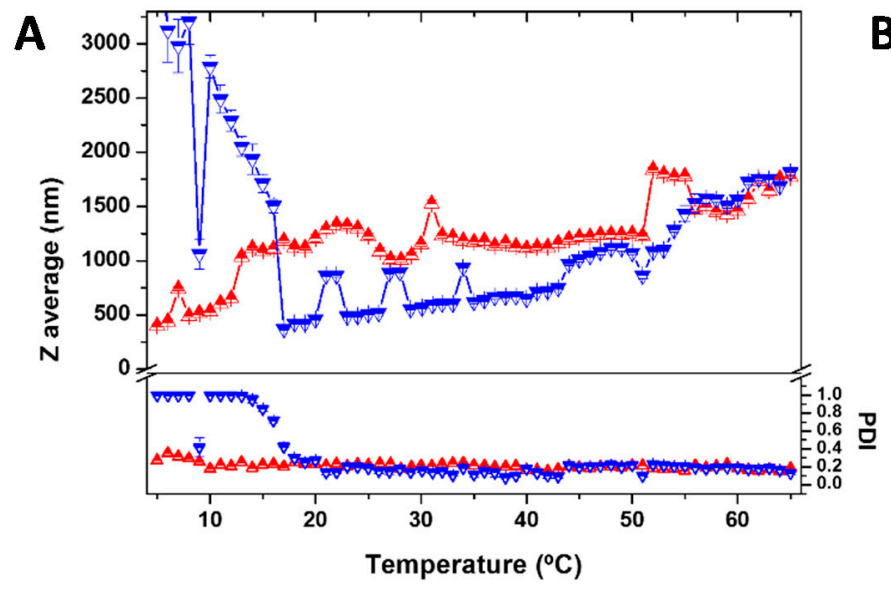

1.0 Eq. CB[7]

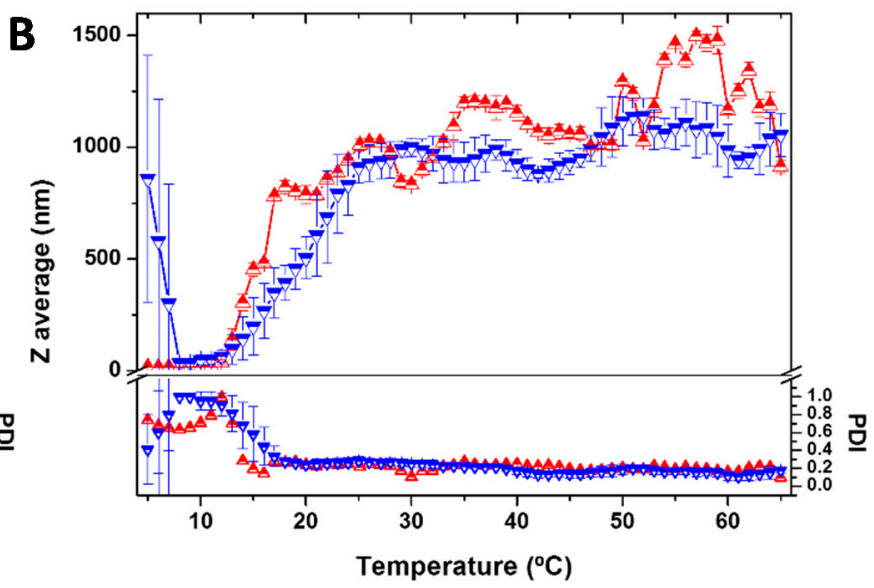

Figure 6. Temperature-dependent Dynamic Light Scattering (DLS) data for a $2 \mathrm{mg} \cdot \mathrm{mL}^{-1}$ solution of $\left.\mathrm{P}\left[(\mathrm{EtOx})_{84} \text {-ran-(NonOx)}\right)_{21}\right]$ in the presence of 0.2 or 1.0 equivalent of $\mathrm{CB} 7$ (relative to nonyl side chains). $\mathrm{Z}$ average and PDI evolution with temperature: >heating; Icooling. (A) 0.2 Eq. CB7: At low temperatures, ill-defined aggregates are formed. Upon heating, macromolecular precipitates appear, that partially re-dissolve upon cooling below $20^{\circ} \mathrm{C}$, resulting in an increase in the $\mathrm{Z}_{\mathrm{ave}}$; (B) $1.0 \mathrm{Eq}$. CB7: At low temperature, random coil ensembles $(\approx 9 \mathrm{~nm})$ coexist with aggregates of sizes from 40 to $300 \mathrm{~nm}$. Beyond $20{ }^{\circ} \mathrm{C}$, large aggregates are formed. Four measurements are averaged at every temperature. Approximate heating/cooling rate: $\approx 0.01 \mathrm{~K} \cdot \mathrm{min}^{-1}$.

To summarize, the increment in NonOx content in the copolymer to $20 \%$ had a strong impact on its solubility properties when compared with the previously studied copolymer with $12 \%$ NonOx. The copolymer was water insoluble and could only be brought in solution in the presence of cavitands. The increased number of nonyl groups per copolymer chain induced the formation of kinetically-trapped 
nanoparticles in solution when sufficiently hydrophilic cavitands, i.e., $\alpha \mathrm{CD}$ and $\mathrm{HP} \alpha \mathrm{CD}$ were present. In this case, the optimal balance of binding affinity to nonyl chains and hydrophilicity was found with native $\alpha \mathrm{CD}$.

\subsection{Highly Hydrophobic Copolymers: Poly(2-ethyl-2-oxazoline)-ran-poly(2-nonyl-2-oxazoline) Containing $25 \%$ and $30 \%$ Nonyl Chains}

In Section 2.2 we have seen that a $20 \%$ content of NonOx in the copolymer began to induce its self-assembly behavior in solution giving rise to kinetically-trapped nanoparticles. To evaluate the possibility to enhance this behavior, two copolymers with the same chain length $(\approx 100$ repeating units) and higher NonOx contents of $25 \%$ and $33 \%$ were synthesized (SN25 and SN33, respectively). The copolymers were dissolved in the presence of $\alpha \mathrm{CD}$ following the same protocol as described for the previous copolymer with $20 \%$ NonOx. Due to the higher hydrophobicity of the newly synthesized copolymers, a higher concentration of $\alpha \mathrm{CD}$ was necessary to bring them in solution. One equivalent of $\alpha \mathrm{CD}$ was required to solubilize SN25, whereas the more hydrophobic SN33 could only be solubilized in a near-saturation concentration of the cavitand. Therefore, the more hydrophilic $\mathrm{HP} \alpha \mathrm{CD}$, with a solubility of over $600 \mathrm{mg} \cdot \mathrm{mL}^{-1}$ was utilized, whereby more than 1.7 equivalents of the cavitand were necessary to solubilize the copolymer. Strong hydrophobic interactions established among the nonyl chains are proposed to hinder the access of the cavitands to establish host-guest complexes that disrupt the polymer-polymer interactions and progressively extend the polymer chain. Host-guest complex formation is thought to open up new nonyl chains for complexation. Thus, repeated cycles of freezing and thawing the solution under sonication at $0{ }^{\circ} \mathrm{C}$ were found to be required to solubilize the copolymer.

Figure 7 shows the results of the titration of both copolymers with $\mathrm{HP} \alpha \mathrm{CD}$. In both cases, addition of more cavitand to the solution had a minor effect on the $\mathrm{T}_{\mathrm{CP}}$. This behavior is ascribed to the formation of kinetically-trapped nanoparticles that contain most of the nonyl chains in the core, isolated from the solution and inaccessible for further host-guest complexation. In the case of SN25, the formation of similar kinetically-trapped nanoparticles as seen for SN20 would explain its homologous thermoresponsive behavior in the presence of $\mathrm{HP} \alpha \mathrm{CD}$.

In analogy with SN20 and SN25, increasing the concentration of $\mathrm{HP} \alpha \mathrm{CD}$ had only a minor effect over the $\mathrm{T}_{\mathrm{CP}}$ of SN33. However, this copolymer exhibited a hysteresis of unprecedented magnitude $(c a .40 \mathrm{~K})$. Once the copolymer-HP $\alpha \mathrm{CD}$ ensemble was dissolved at low temperatures, the solution remained transparent up to $c a .50^{\circ} \mathrm{C}$, when the solution became white opaque due to aggregation of copolymer-HP $\alpha \mathrm{CD}$ nanoparticles. The solution then remained opaque in the whole temperature range, and it was necessary to lower the temperature to $c a .0{ }^{\circ} \mathrm{C}$ to break the aggregates and recover a transparent solution containing individual nanoparticles. The copolymer-HP $\alpha \mathrm{CD}$ ensemble was thus able to remember the thermal history of the solution, resulting in a temperature sensor with memory function. An in-depth study was performed to understand the reasons behind the highly unusual solution behavior of this copolymer-cavitand ensemble and has been reported elsewhere [43]. It is thus apparent that increasing the content of hydrophobic nonyl chains in the copolymer further increases the stability of the kinetically-trapped nanoparticles. These nanoparticles only expose a minor fraction of nonyl chains to the surrounding medium, directing most nonyl chains into the hydrophobic core of the nanoparticle. 

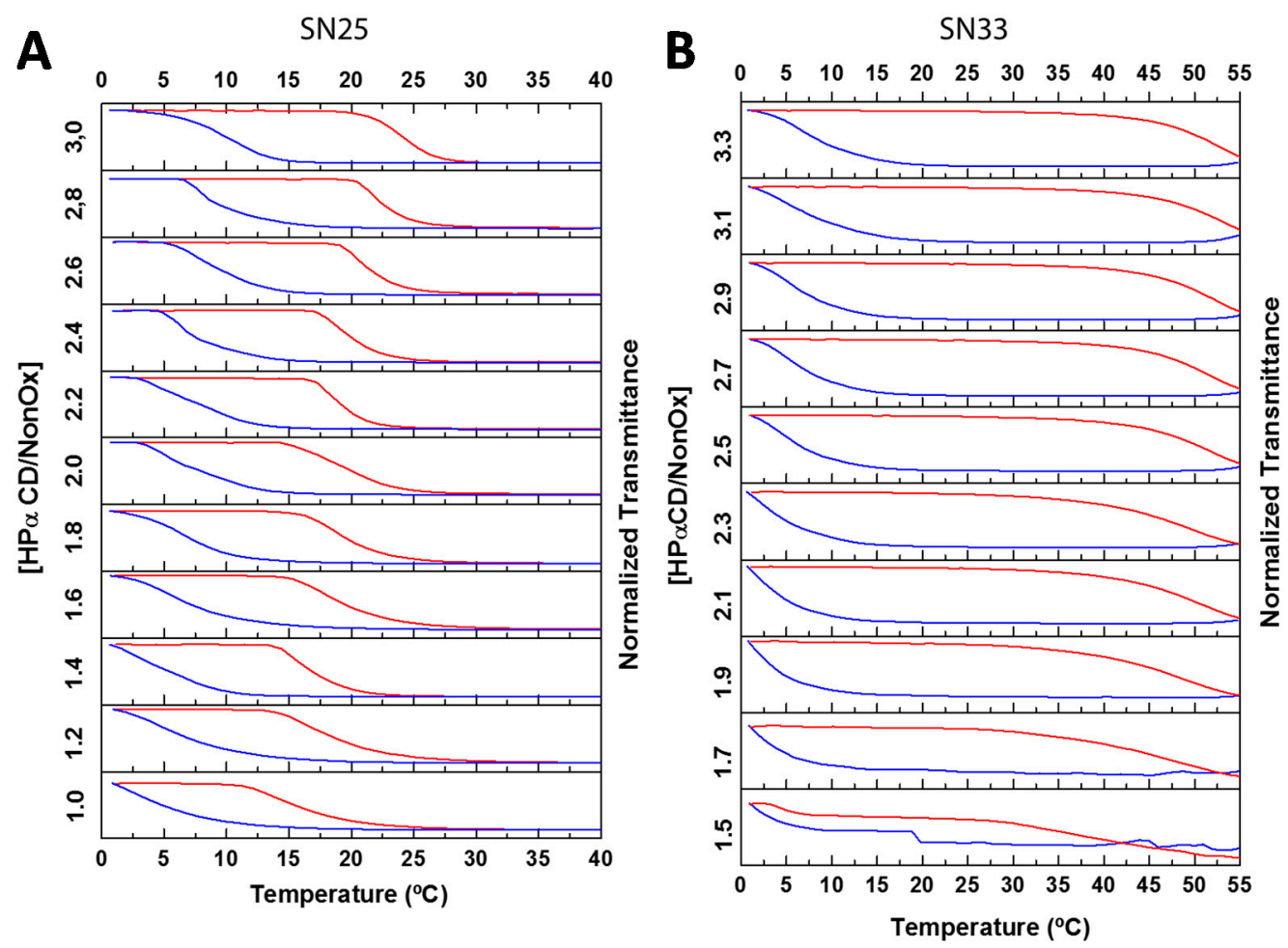

Figure 7. Turbidimetry studies of of $5 \mathrm{mg} \cdot \mathrm{mL}^{-1}$ solutions of $\mathrm{P}\left[(\mathrm{EtOx})_{75}-\mathrm{ran}-(\mathrm{NonOx})_{25}\right]$ (A, SN25) and P[(EtOx)62-ran-(NonOx)29] (B, SN33) with increasing HPaCD/NonOx molar ratios. Both copolymers exhibited minor variations of the $\mathrm{T}_{\mathrm{CP}}$ upon addition of increasing amounts of cavitand, which is ascribed to nanoparticle formation where the nonyl chains are isolated from the aqueous environment. In the case of SN33, an unprecedented large hysteresis of $40{ }^{\circ} \mathrm{C}$ was found. The heating and cooling ramps are represented by the top (red) and bottom (blue) curves, respectively. Rate: $1 \mathrm{~K} \cdot \mathrm{min}^{-1}, \lambda=700 \mathrm{~nm}$.

\subsection{Poly(2-ethyl-2-oxazoline)-ran-poly(2-nonyl-2-oxazoline): Influence of Polymer Length}

Finally, the effect of polymer chain length on the solubility behavior of the PEtOx-ran-PNonOx copolymers was investigated. To this end, two copolymers were synthesized bearing $c a$. 200 repeating units, thus double the length of the copolymers studied previously. The copolymers had a NonOx content of $19 \%$ (LN19) or 29\% (LN29) and were, as expected, insoluble in the absence of cavitands. In fact, the increase in polymer chain length rendered, as expected, both copolymers more difficult to dissolve, requiring 2 and 4 equivalents of $\mathrm{HP} \alpha \mathrm{CD}$ to solubilize LN19 and LN29, respectively.

Figure 8 shows the temperature dependent turbidimetry results, which yielded a sharp LCST transition in both copolymers, in contrast with the more progressive transition found in the shorter copolymers in the presence of $\mathrm{HP} \alpha \mathrm{CD}$. The $\mathrm{T}_{\mathrm{CP}}$ of $\mathrm{LN} 19$ only increased by $c a .5 \mathrm{~K}$ when increasing the amount of the cavitand from 2 to 4 equivalents, therefore indicating the unavailability of most of the copolymer nonyl chains to engage in host-guest complexation. The higher number of nonyl groups present in a single polymer chain thus seems to enhance the intramolecular nonyl-nonyl hydrophobic interactions further stabilizing the insoluble globular morphology of the copolymer. This results in the need to add a large excess of $\mathrm{HP} \alpha \mathrm{CD}$ to disrupt the nonyl-nonyl hydrophobic interactions and is most evident in the case of LN29, which requires up to 4 equivalents of $\mathrm{HP} \alpha \mathrm{CD}$ to be solubilized. 

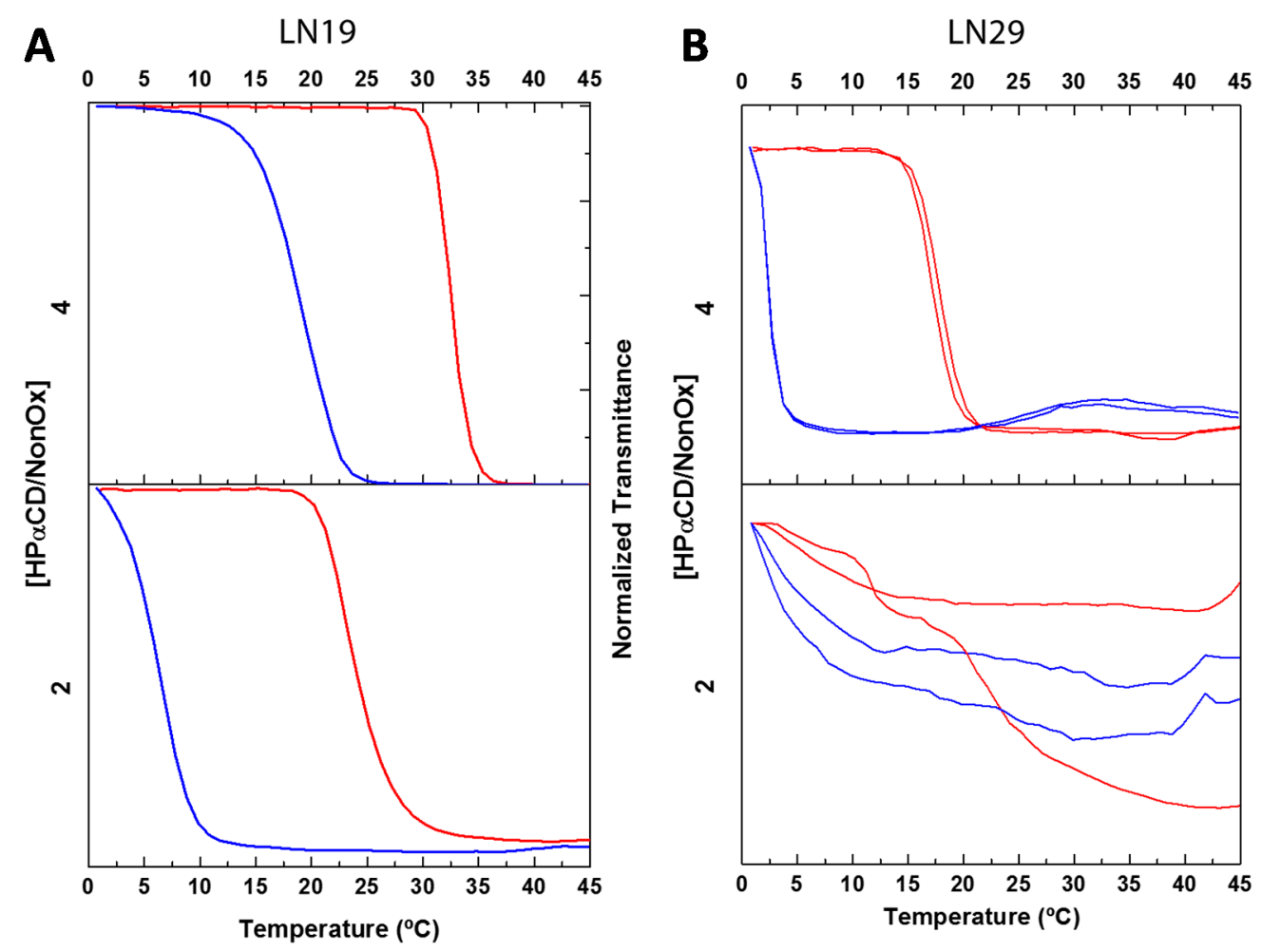

Figure 8. Turbidimetry studies of $5 \mathrm{mg} \cdot \mathrm{mL}^{-1}$ solutions of $\mathrm{P}\left[(\mathrm{EtOx})_{162}-\right.$ ran-(NonOx) 38$]$ (A, LN19) and P[(EtOx) 140 -ran-(NonOx)57] (B, LN29) with increasing HPaCD/NonOx molar ratios. Large $\mathrm{HP} \alpha \mathrm{CD}$ excesses are required to solubilize both copolymers, especially in the case of LN29. The heating and cooling ramps are represented by the top (red) and bottom (blue) curves, respectively. Rate: $1 \mathrm{~K} \cdot \mathrm{min}^{-1}, \lambda=700 \mathrm{~nm}$.

\section{Experimental Section}

\subsection{Materials}

Solvents and reagents were purchased from Sigma Aldrich (St. Louis, MO, USA), and used as received unless otherwise specified. Cucurbit[7]uril was synthesized as reported [49,50]. Methyl tosylate (MeOTs) was distilled twice under vacuum prior to use. 2-Ethyl-2-oxazoline (EtOx), 2-nonyl-2-oxazoline (NonOx, Henkel, Dusseldorf, Germany), were distilled over barium oxide (BaO). Acetonitrile $\left(\mathrm{CH}_{3} \mathrm{CN}\right.$, Acros Organics, Geel, Belgium) was dried over molecular sieves ( $\left.3 \AA\right)$. All reagents were stored and handled under a dry argon or nitrogen atmosphere.

Deionized (Milli-Q) water was obtained from a Sartorius Arium 611 with a Sartopore 2150 $(0.45+0.2 \mu \mathrm{m}$ pore size) cartridge filter (resistivity $\geq 18.2 \mathrm{M} \Omega \cdot \mathrm{cm})$ (Sartorius, Göttingen, Germany)

\subsection{Instrumentation}

The polymerization was performed in a Biotage initiator sixty microwave synthesizer (Biotage, Uppsala, Sweden) utilizing capped microwave vials. The vials were heated to $120{ }^{\circ} \mathrm{C}$ for $24 \mathrm{~h}$ and cooled down to room temperature under vacuum prior to use. The polymerization was performed with temperature control (IR sensor) 
${ }^{1} \mathrm{H}-\mathrm{NMR}$ spectroscopy was performed in $\mathrm{CDCl}_{3}$ on a Bruker Avance $300 \mathrm{MHz}$ spectrometer (Bruker Corporation, Billerica, MA, USA). Spectra were processed using TOPSPIN 3.0 (Bruker Corporation, Billerica, MA, USA).

Size exclusion chromatography (SEC) measurements were performed on an Agilent 1260 -series equipped with a 1260 ISO-pump, a 1260 Diode Array Detector (DAD), a 1260 Refractive Index Detector (RID), and two Mixed-D columns and a Mixed-D guard column (Agilent Technologies, Santa Clara, CA, USA) in series inside a 1260 Thermostated Column Compartment (TCC) at $50{ }^{\circ} \mathrm{C}$ using dimethylacetamide containing $50 \mathrm{mM}$ of $\mathrm{LiCl}$ (flow rate of $0.6 \mathrm{~mL} \cdot \mathrm{min}^{-1}$ ) as solvent. Molar mass and dispersity were calculated against poly(methyl methacrylate) standards.

\subsection{Turbidimetry and Dynamic Light Scattering Studies}

Turbidimetry measurements were performed in a CARY Bio $100 \mathrm{UV}-\mathrm{VIS}$ spectrophotometer equipped (Agilent Technologies, Santa Clara, CA, USA) with a temperature controller, at a wavelength of $700 \mathrm{~nm}$. Heating/cooling cycles were performed at a rate of $1 \mathrm{~K} \cdot \mathrm{min}^{-1}$ with stirring. The polymer concentration was kept at $5 \mathrm{mg} \cdot \mathrm{mL}^{-1}$ in deionized water. The equivalents of cyclodextrin and cucurbit[7]uril added were calculated in relation to the equivalents of nonyl side chains.

\subsection{Poly[(2-ethyl-2-oxazoline)-ran-(2-nonyl-2-oxazoline)] Synthesis}

The polymerizations were performed as previously reported [26]. The microwave vials were loaded in a glove box (Vigor Gas Purification Technologies Inc., Houston, TX, USA) with EtOx and NonOx copolymers in the desired molar ratio, maintaining a total monomer concentration of $4 \mathrm{M}$ in acetonitrile. Polymer length was tuned by the ratio of MeOTs initiator to monomer. Table 2 summarizes the amounts of initiator, monomers, and solvent that were used to produce each copolymer.

The polymerizations were run for $15 \mathrm{~min}$ at $140{ }^{\circ} \mathrm{C}$ (DP 100) or $30 \mathrm{~min}$ (DP 200). The polymers were terminated with $\mathrm{KOH}$ in methanol, yielding hydroxyl-terminated polymers. The solvent was evaporated under reduced pressure, and each polymer was subsequently precipitated in diethyl ether from dichloromethane. The pure polymer was then dried in a vacuum oven at $50{ }^{\circ} \mathrm{C}$ for $24 \mathrm{~h}$.

Table 2. Reagents quantities and obtained copolymer compositions.

\begin{tabular}{|c|c|c|c|c|c|c|c|}
\hline ID & $\begin{array}{l}\text { Target Polymer } \\
\text { Composition }\end{array}$ & $\begin{array}{c}\text { Obtained Polymer } \\
\text { Composition }^{\text {(a) }}\end{array}$ & $\begin{array}{c}\text { DP } \\
\text { (Target/Obtained) }\end{array}$ & $\begin{array}{c}\text { MeOTs } \\
\text { (g) }\end{array}$ & $\begin{array}{l}\text { EtOx } \\
(g)\end{array}$ & $\begin{array}{c}\text { NonOx } \\
\text { (g) }\end{array}$ & $\begin{array}{r}\mathrm{MeCN} \\
(\mathrm{mL})\end{array}$ \\
\hline SN12 & $\mathrm{PEtOx}_{90}-r a n-\mathrm{PNonO}_{10}$ & PEtOx$_{90}-$ ran-PNonOx 12 & $100 / 102$ & 0.0335 & 1.606 & 0.355 & 2.491 \\
\hline SN20 & $\mathrm{PEtOx}_{80}-r a n-\mathrm{PNonO}_{20}$ & PEtOx$_{84-r a n-P N o n O x_{21}}$ & $100 / 105$ & 0.0272 & 1.454 & 0.747 & 2.299 \\
\hline SN25 & $\mathrm{PEtOx}_{75}$-ran-PNonOx 25 & $\mathrm{PEtOx}_{75}$-ran-PNonOx 25 & $100 / 100$ & 0.0223 & 0.892 & 0.592 & 1.155 \\
\hline SN33 & $\mathrm{PEtOx}_{70}$-ran-PNonOx 30 & $\mathrm{PEtOx}_{62}$-ran-PNonOx 29 & $100 / 91$ & 0.0272 & 1.272 & 1.120 & 2.108 \\
\hline LN19 & $\mathrm{PEtOx}_{160}$-ran-PNonOx 40 & $\mathrm{PEtOx}_{162}$-ran-PNonOx 38 & $200 / 200$ & 0.0109 & 0.933 & 0.464 & 1.502 \\
\hline LN29 & PEtOx $140-r a n-P N o n O x 60$ & PEtOx140-ran-PNonOx57 & $200 / 197$ & 0.0112 & 0.833 & 0.710 & 1.104 \\
\hline
\end{tabular}

\footnotetext{
${ }^{\text {a }}$ Calculated by ${ }^{1} \mathrm{H}-\mathrm{NMR}$ spectroscopy.
} 


\subsection{Preparation of Poly[(2-ethyl-2-oxazoline)-ran-(2-nonyl-2-oxazoline)] Solutions and Titration}

For all the titrations with a cavitand stock solution, PMMA cuvettes for Vis-spectroscopy (Carl-Roth), each equipped with a stirring bar, were filled with $2 \mathrm{~mL}$ of copolymer solution. To calculate the amount of cavitand stock solution necessary to add to each cuvette, the following calculations were performed.

First, the weight fraction of $\mathrm{NonOx}\left(f_{\mathrm{NonOx}}\right)$ in the copolymer was calculated, according to the following equation:

$$
\text { Wt. fraction NonOx }\left(f_{\text {NonOx }}\right)=\frac{D P(\mathrm{NonOx}) M_{w t}(\mathrm{NonOx})}{D P(\text { EtOx }) M_{w t}(\mathrm{EtOx})+D P(\text { NonOx }) M_{w t}(\text { NonOx })}
$$

where $D P$ is the degree of polymerization i.e., the number of repeating units of each monomer in the copolymer.

Then, the mass of cavitand necessary to equal the number of NonOx groups contained in $2 \mathrm{~mL}$ (cuvette) of $5 \mathrm{mg} \cdot \mathrm{mL}^{-1}$ copolymer solution is calculated.

$$
m \text { cavitand }(\text { grams })=\frac{f_{\mathrm{NonOx}} \times 5 \mathrm{mg} \mathrm{mL}^{-1} \times 2 \mathrm{~mL}}{M_{w t}(\mathrm{NonOx})} \times M_{w t}(\text { cavitand })
$$

Finally, the volume of cavitand stock solution required to add to each copolymer solution is calculated:

$$
V \text { cavitand }(\mu \mathrm{L})=1000 \times \frac{m \text { cavitand }(\mathrm{g})}{\text { concentration cavitand }\left(\frac{\mathrm{g}}{\mathrm{mL}}\right)} \times \text { Number of equivalents }
$$

In the present study, the number of equivalents ranged from 0.2 to 2.0 . The aliquots were measured and dispensed with a micropipette.

Since the investigated copolymers were insoluble, a $5 \mathrm{mg} \cdot \mathrm{mL}^{-1}$ stock solution was prepared in the presence of cavitand (e.g., 0.2 equivalents $\mathrm{HP} \alpha \mathrm{CD}$ for SN20, or 1.7 equivalents $\mathrm{HP} \alpha \mathrm{CD}$ for SN33). This cavitand-copolymer mixture was frozen and thawed under sonication in an ice bath. This protocol was repeated until all macroscopic polymer particles disappeared.

PMMA cuvettes were filled with $2 \mathrm{~mL}$ of the copolymer solution. Subsequently, increasing amounts of the cavitand stock solution were added to each cuvette, obtaining the desired cavitand concentration. Cavitand stock solution concentrations ranged from $120 \mathrm{mg} \cdot \mathrm{mL}^{-1}$ for $\alpha \mathrm{CD}$ to $150 \mathrm{mg} \cdot \mathrm{mL}^{-1}$ for $\mathrm{HP} \alpha \mathrm{CD}$. The dilution of the copolymer dilution by addition of cavitand was always kept below $10 \%$.

Due to the relatively low solubility of CB7, a $4 \mathrm{mM}$ solution of the cavitand was prepared $\left(4.83 \mathrm{mg} \cdot \mathrm{mL}^{-1}\right)$. To avoid high dilution effects upon titration, the copolymer was directly weighted in the cuvette, and the necessary amounts of water and CB7 stock solution were added to obtain a $5 \mathrm{mg} \cdot \mathrm{mL}^{-1}$ concentration of copolymer.

\section{Conclusions}

The copolymer composition of PEtOx-ran-PNonOx copolymers was found to exert a tremendous impact on its host-guest complexation capabilities and on the structures formed at the nanoscale upon complexation with supramolecular hosts. In our previous study comprising a PEtOx-ran-PNonOx copolymer containing 12\% NonOx, cavitand-nonyl complexation resulted in the extension of the 
polymer chain and the formation of random-coil polymer-cavitand ensembles. Increasing the nonyl content to $20 \%-25 \%$ promoted the formation of kinetically-trapped nanoparticles, in which the core nonyl chains are shielded from the aqueous environment. Macroscopically, this translated into a progressive temperature-induced phase transition. When the NonOx content was further increased to $33 \%$ kinetically-trapped nanoparticle formation promoted the appearance of a large hysteresis, which resulted in temperature sensors with the potential to exhibit a memory function [43].

Finally, increasing the copolymer chain length to 200 repeating units seemed to favor intramolecular nonyl-nonyl hydrophobic interactions, promoting a polymer globular structure and hindering host-guest complex formation. Thus, large excess of cavitands was required to solubilize these longer copolymers.

Overall, the results obtained demonstrate that, when coupled to a polymer structure, simple and relatively weak supramolecular interactions in water can trigger the formation of a variety of complex, defined architectures at the nanoscale. These supramolecular architectures are determined by subtle changes in molecular structure and dictate the macroscopic behavior of the solution. The larger number of hydrophobic units in water insoluble PEtOx-ran-PNonOx copolymers in combination with highly hydrophilic supramolecular hosts, favored the formation of well-defined kinetically-trapped nanoparticles.

Besides advancing in the understanding of the interplay between amphiphilic polymers and supramolecular host molecules, further research could be directed to the use of the obtained nanoparticles in the solubilization of hydrophobic molecules of relevance in biomedical applications.

\section{Acknowledgments}

Richard Hoogenboom and Victor R. de la Rosa would like to thank Ghent University for financial support through the Concerted Research Actions (project BOF11/GOA/023) and the Fund for Scientific Research-Flanders (FWO) for support through the Scientific Research Network (WOG) on Supramolecular Chemistry. The authors also thank the COST Action CM 1005 "Supramolecular Chemistry in Water".

\section{Author Contributions}

Victor R. de la Rosa synthesized the copolymers and performed the experiments. Werner M. Nau provided CB7. Richard Hoogenboom supervised the research. All were involved in drafting and correcting the manuscript.

\section{Conflicts of Interest}

The authors declare no conflict of interest.

\section{References}

1. Zhang, J.; Pu, G.; Dubay, M.R.; Zhao, Y.; Severtson, S.J. Repositionable pressure-sensitive adhesive possessing thermal-stimuli switchable transparency. J. Mater. Chem. C 2013, 1, 1080-1086.

2. Rotzetter, A.C.; Schumacher, C.M.; Bubenhofer, S.B.; Grass, R.N.; Gerber, L.C.; Zeltner, M.; Stark, W.J. Thermoresponsive polymer induced sweating surfaces as an efficient way to passively cool buildings. Adv. Mater. 2012, 24, 5352-5356. 
3. Yang, H.; Zhu, H.; Hendrix, M.M.R.M.; Lousberg, N.J.H.G.M.; de With, G.; Esteves, A.C.C.; Xin, J.H. Temperature-triggered collection and release of water from fogs by a sponge-like cotton fabric. Adv. Mater. 2013, 25, 1150-1154.

4. Kikuchi, A.; Okano, T. Intelligent thermoresponsive polymeric stationary phases for aqueous chromatography of biological compounds. Prog. Polym. Sci. 2002, 27, 1165-1193.

5. Tan, I.; Roohi, F.; Titirici, M.-M. Thermoresponsive polymers in liquid chromatography. Anal. Methods 2011, 4, 34-43.

6. Defize, T.; Riva, R.; Raquez, J.-M.; Dubois, P.; Jérôme, C.; Alexandre, M. Thermoreversibly crosslinked poly(E-caprolactone) as recyclable shape-memory polymer network. Macromol. Rapid Commun. 2011, $32,1264-1269$.

7. Klouda, L.; Mikos, A.G. Thermoresponsive hydrogels in biomedical applications. Eur. J. Pharm. Biopharm. 2008, 68, 34-45.

8. Ward, M.A.; Georgiou, T.K. Thermoresponsive polymers for biomedical applications. Polymers 2011, 3, 1215-1242.

9. Kedar, U.; Phutane, P.; Shidhaye, S.; Kadam, V. Advances in polymeric micelles for drug delivery and tumor targeting. Nanomed. NBM 2010, 6, 714-729.

10. Brun-Graeppi, A.K.A.S.; Richard, C.; Bessodes, M.; Scherman, D.; Merten, O.-W. Thermoresponsive surfaces for cell culture and enzyme-free cell detachment. Prog. Polym. Sci. 2010, 35, 1311-1324.

11. Lee, J.; Kotov, N.A. Thermometer design at the nanoscale. Nano Today 2007, 2, 48-51.

12. Gota, C.; Okabe, K.; Funatsu, T.; Harada, Y.; Uchiyama, S. Hydrophilic fluorescent nanogel thermometer for intracellular thermometry. J. Am. Chem. Soc. 2009, 131, 2766-2767.

13. McCabe, K.M.; Hernandez, M. Molecular thermometry. Pediatr. Res. 2010, 67, 469-475.

14. Pietsch, C.; Schubert, U.S.; Hoogenboom, R. Aqueous polymeric sensors based on temperature-induced polymer phase transitions and solvatochromic dyes. Chem. Commun. 2011, 47, 8750-8765.

15. Pietsch, C.; Vollrath, A.; Hoogenboom, R.; Schubert, U.S. A fluorescent thermometer based on a pyrene-labeled thermoresponsive polymer. Sensors 2010, 10, 7979-7990.

16. Okabe, K.; Inada, N.; Gota, C.; Harada, Y.; Funatsu, T.; Uchiyama, S. Intracellular temperature mapping with a fluorescent polymeric thermometer and fluorescence lifetime imaging microscopy. Nat. Commun. 2012, 3, 705.

17. Hoogenboom, R. Smart Polymers and their Applications; Aguilar, M.R., Román, J.S., Eds.; Woodhead Publishing: Cambridge, UK, 2014; pp. 15-44.

18. Lutz, J.-F. Thermo-switchable materials prepared using the OEGMA-platform. Adv. Mater. 2011, $23,2237-2243$.

19. Eggenhuisen, T.M.; Becer, C.R.; Fijten, M.W.M.; Eckardt, R.; Hoogenboom, R.; Schubert, U.S. Libraries of statistical hydroxypropyl acrylate containing copolymers with LCST properties prepared by NMP. Macromolecules 2008, 41, 5132-5140.

20. Glassner, M.; Lava, K.; de la Rosa, V.R.; Hoogenboom, R. Tuning the LCST of poly(2-cyclopropyl-2-oxazoline) via gradient copolymerization with 2-ethyl-2-oxazoline. J. Polym. Sci. Part A Polym. Chem. 2014, 52, 3118-3122.

21. Huber, S.; Jordan, R. Modulation of the lower critical solution temperature of 2-alkyl-2-oxazoline copolymers. Colloid Polym. Sci. 2008, 286, 395-402. 
22. Diehl, C.; Schlaad, H. Thermo-responsive polyoxazolines with widely tuneable LCST. Macromol. Biosci. 2009, 9, 157-161.

23. Park, J.-S.; Kataoka, K. Comprehensive and accurate control of thermosensitivity of poly(2-alkyl-2-oxazoline)s via well-defined gradient or random copolymerization. Macromolecules 2007, 40, 3599-3609.

24. Park, J.-S.; Kataoka, K. Precise control of lower critical solution temperature of thermosensitive poly(2-isopropyl-2-oxazoline) via gradient copolymerization with 2-ethyl-2-oxazoline as a hydrophilic comonomer. Macromolecules 2006, 39, 6622-6630.

25. Hoogenboom, R.; Thijs, H.M.L.; Jochems, M.J.H.C.; van Lankvelt, B.M.; Fijten, M.W.M.; Schubert, U.S. Tuning the LCST of poly(2-oxazoline)s by varying composition and molecular weight: Alternatives to poly( $N$-isopropylacrylamide)? Chem. Commun. 2008, 2008, 5758-5760.

26. Fijten, M.W.M.; Kranenburg, J.M.; Thijs, H.M.L.; Paulus, R.M.; van Lankvelt, B.M.; de Hullu, J.; Springintveld, M.; Thielen, D.J.G.; Tweedie, C.A.; Hoogenboom, R.; et al. Synthesis and structure-property relationships of random and block copolymers: A direct comparison for copoly(2-oxazoline)s. Macromolecules 2007, 40, 5879-5886.

27. Lambermont-Thijs, H.M. L.; Hoogenboom, R.; Fustin, C.-A.; Bomal-D’Haese, C.; Gohy, J.-F.; Schubert, U.S. Solubility behavior of amphiphilic block and random copolymers based on 2-ethyl-2-oxazoline and 2-nonyl-2-oxazoline in binary water-ethanol mixtures. J. Polym. Sci. Part A Polym. Chem. 2009, 47, 515-522.

28. Volet, G.; Chanthavong, V.; Wintgens, V.; Amiel, C. Synthesis of monoalkyl end-capped poly(2-methyl-2-oxazoline) and its micelle formation in aqueous solution. Macromolecules 2005, $38,5190-5197$.

29. Ritter, H.; Sadowski, O.; Tepper, E. Influence of cyclodextrin molecules on the synthesis and the thermoresponsive solution behavior of $N$-isopropylacrylamide copolymers with adamantyl groups in the side-chains. Angew. Chem. Int. Ed. 2003, 42, 3171-3173.

30. Schmidt, B.V.K.J.; Hetzer, M.; Ritter, H.; Barner-Kowollik, C. Modulation of the thermoresponsive behavior of poly $(N, N$-diethylacrylamide $)$ via cyclodextrin host/guest interactions. Macromol. Rapid Commun. 2013, 34, 1306-1311.

31. Reinelt, S.; Steinke, D.; Ritter, H. End-group-functionalized poly $(N, N$-diethylacrylamide $)$ via free-radical chain transfer polymerization: Influence of sulfur oxidation and cyclodextrin on self-organization and cloud points in water. Beilstein J. Org. Chem. 2014, 10, 680-691.

32. Burkhart, A.; Ritter, H. Influence of cyclodextrin on the UCST- and LCST-behavior of poly(2-methacrylamido-caprolactam)-co-( $N, N$-dimethylacrylamide). Beilstein J. Org. Chem. 2014, 10, 1951-1958.

33. Sambe, L.; de la Rosa, V.R.; Belal, K.; Stoffelbach, F.; Lyskawa, J.; Delattre, F.; Bria, M.; Cooke, G.; Hoogenboom, R.; Woisel, P. Programmable polymer-based supramolecular temperature sensor with a memory function. Angew. Chem. Int. Ed. 2014, 53, 5044-5048.

34. Sakai, R.; Otsuka, I.; Satoh, T.; Kakuchi, R.; Kaga, H.; Kakuchi, T. Thermoresponsive on-off switching of chiroptical property induced in poly(4'-ethynylbenzo-15-crown-5)/ $\alpha$-amino acid system. Macromolecules 2006, 39, 4032-4037.

35. Yashima, E.; Maeda, K.; Sato, O. Switching of a macromolecular helicity for visual distinction of molecular recognition events. J. Am. Chem. Soc. 2001, 123, 8159-8160. 
36. Bigot, J.; Charleux, B.; Cooke, G.; Delattre, F.; Fournier, D.; Lyskawa, J.; Sambe, L.; Stoffelbach, F.; Woisel, P. Tetrathiafulvalene end-functionalized poly( $N$-isopropylacrylamide): A new class of amphiphilic polymer for the creation of multistimuli responsive micelles. J. Am. Chem. Soc. 2010 , 132, 10796-10801.

37. Sambe, L.; Stoffelbach, F.; Lyskawa, J.; Delattre, F.; Fournier, D.; Bouteiller, L.; Charleux, B.; Cooke, G.; Woisel, P. Host-guest modulation of the micellization of a tetrathiafulvalene-functionalized poly( $N$-isopropylacrylamide). Macromolecules 2011, 44, 6532-6538.

38. Chi, X.; Ji, X.; Xia, D.; Huang, F. A Dual-responsive supra-amphiphilic polypseudorotaxane constructed from a water-soluble pillar[7] arene and an azobenzene-containing random copolymer. J. Am. Chem. Soc. 2015, 137, 1440-1443.

39. Yu, G.; Jie, K.; Huang, F. Supramolecular amphiphiles based on host-guest molecular recognition motifs. Chem. Rev. 2015, doi:10.1021/cr5005315.

40. Ji, X.; Li, J.; Chen, J.; Chi, X.; Zhu, K.; Yan, X.; Zhang, M.; Huang, F. Supramolecular micelles constructed by crown ether-based molecular recognition. Macromolecules 2012, 45, 6457-6463.

41. Ji, X.; Dong, S.; Wei, P.; Xia, D.; Huang, F. A novel diblock copolymer with a supramolecular polymer block and a traditional polymer block: Preparation, controllable self-assembly in water, and application in controlled release. Adv. Mater. 2013, 25, 5725-5729.

42. De la Rosa, V.R.; Nau, W.M.; Hoogenboom, R. Tuning temperature responsive poly(2-alkyl-2oxazoline)s by supramolecular host-guest interactions. Org. Biomol. Chem. 2015, 13, 3048-3057.

43. De la Rosa, V.R.; Hoogenboom, R. Solution polymeric optical temperature sensors with long-term memory function powered by supramolecular chemistry. Chem. Eur. J. 2015, 21, 1302-1311.

44. Glassner, M.; D’hooge, D.R.; Park, J.Y.; van Steenberge, P.H.M.; Monnery, B.D.; Reyniers, M.-F.; Hoogenboom, R. Systematic investigation of alkyl sulfonate initiators for the cationic ring-opening polymerization of 2-oxazolines revealing optimal combinations of monomers and initiators. Eur. Polym. J. 2015, 65, 298-304.

45. Hoogenboom, R.; Fijten, M.W.M.; Wijnans, S.; van den Berg, A.M.J.; Thijs, H.M.L.; Schubert, U.S. High-throughput synthesis and screening of a library of random and gradient copoly(2-oxazoline)s. J. Comb. Chem. 2006, 8, 145-148.

46. Litt, M.; Levy, A.; Herz, J. Polymerization of cyclic imino ethers. X. Kinetics, chain transfer, and repolymerization. J. Macromol. Sci. Chem. 1975, 9, 703-727.

47. Lagona, J.; Mukhopadhyay, P.; Chakrabarti, S.; Isaacs, L. The cucurbit[n]uril family. Angew. Chem. Int. Ed. 2005, 44, 4844-4870.

48. Szejtli, J. Introduction and general overview of cyclodextrin chemistry. Chem. Rev. 1998, 98, 1743-1754.

49. Kim, J.; Jung, I.-S.; Kim, S.-Y.; Lee, E.; Kang, J.-K.; Sakamoto, S.; Yamaguchi, K.; Kim, K. New cucurbituril homologues: Syntheses, isolation, characterization, and X-ray crystal structures of cucurbit $[n]$ uril ( $n=5,7$, and 8). J. Am. Chem. Soc. 2000, 122, 540-541.

50. Day, A.; Arnold, A.P.; Blanch, R.J.; Snushall, B. Controlling factors in the synthesis of cucurbituril and its homologues. J. Org. Chem. 2001, 66, 8094-8100.

(C) 2015 by the authors; licensee MDPI, Basel, Switzerland. This article is an open access article distributed under the terms and conditions of the Creative Commons Attribution license (http://creativecommons.org/licenses/by/4.0/). 